\title{
Introduction to Special Issue
}

\section{Children and Youth with Traumatic Brain Injury}

\author{
Marilyn Lash* \\ Lash and Associates Publishing/Training, Inc., Wake Forest, NC, USA
}

The special needs of children and youth after traumatic brain injury are slowly gaining greater recognition and appreciation in medical and rehabilitation environments, as well as in schools and the community. Yet, while great advances have been made in the early stages of trauma care and treatment for this population, we still do not have a comprehensive understanding of the long-term consequences of traumatic brain injury for the child's development and achievement. Nor do we have a solid body of research and programs specific to traumatic brain injury in childhood that can help families and educators guide the adolescent's transition into adulthood.

Families and educators are particularly challenged as they become the long-term caregivers and providers in the community. Despite the landmark inclusion of traumatic brain injury as a category for students under the Individuals with Disabilities Education Act in 1991, there is still a low incidence of educational reporting in all states. This contrasts to the medical data which report that traumatic brain injury is the leading cause of disability in children and youth.

Witnessing their child's frustration at school and altered relationships with siblings and peers can be isolating and frustrating for families as they seek help. The inexperience and limited training regarding brain injury of many professionals in local schools and com-

* Address for correspondence: Marilyn Lash, M.S.W., Lash and Associates Publishing/Training, Inc., 708 Young Forest Drive, Wake Forest, NC 27587, USA. Tel./Fax: +1 919562 0015; E-mail: mlyn@lapublishing.com. munity agencies compound the cumulative stresses on these parents as they seek guidance and expertise to help them understand how the brain injury has affected their son or daughter.

For these reasons, this special issue on children and youth focuses on life after the initial hospital stay and concentrates on the challenges in school and the community. The challenge of providing rehabilitation to school age children is incredibly complex because so many of the social, emotional, behavioral and cognitive issues become evident at home and in school over time.

Dr. Steven Hooper and his colleagues start with perhaps the most important source of information - the parents - by examining caregiver reports of common symptoms in children following a traumatic brain injury. The importance of this article is the 3 stage follow up at 1, 4 and 10 month intervals after discharge. Examining differences between children released from emergency departments and those admitted to the hospital sheds valuable data on persistent symptoms and difficulties at school and home over the first year postinjury.

Our understanding of the long-term outcomes of children and youth with brain injury has been hampered by the difficulty of developing measures for this population. Many of the instruments used in research studies involving adults with traumatic brain injury are simply not appropriate for children. There is a minimum age requirement of 16 years for inclusion in research under the Traumatic Brain Injury Model Systems programs. Unfortunately, there is no comparable body of research specifically for children and youth with brain 
injury. While the National Pediatric Trauma Registry provided extensive data on the trauma care and treatment of this population, it captured no follow-up data after discharge.

Dr. Gary Bedell addresses this need and challenge in his article which describes the development of measures of participation, environment and child factors for children and youth with acquired brain injuries. These were designed to monitor the needs and outcomes of children as well as their families after discharge from inpatient rehabilitation.

The challenges of interfacing with school systems and building the capacity of educators to recognize and respond to the needs of students with brain injuries are presented in two articles based on innovative models in Canada and the United States. Dr. Jane Gillett describes the Pediatric Acquired Brain Injury Community Outreach Program in Ontario and Dr. Ann Glang and her colleagues present the Traumatic Brain Injury Team model of statewide consulting teams in Iowa, Kansas and Oregon.

The challenges and opportunities for using technology offer both promise and confusion with the continuing development and sophistication of devices and software. Drs. Yvonne Gillette and Roberta DePompei analyze the potential of electronic organizers as a tool in the cognitive rehabilitation of young people. The inclusion of youths, parents and teachers in the study provides useful insights from differing perspectives as does the inclusion of youths with traumatic brain injury as well as mental retardation and other developmental disabilities.

Finally, the challenge of brain injury during the teen years is addressed by Dr. Lyn Turkstra and colleagues in a discussion of Theory of Mind and social beliefs in adolescents with traumatic brain injury. During this stage of maturation, social cognition becomes increasingly critical for the development of relationships and preparation for adulthood.

Hopefully, this issue will provide you with greater knowledge and understanding about the challenges faced by children and youth and their families as they embark on the long journey home after brain injury. We are truly just at the beginning of understanding how a traumatic injury can affect the development and functioning of the child's brain. Whether you are a clinician, educator, researcher, policy analyst or advocate, we each must share some responsibility and commit to making a difference in the lives of these children and their families because it really does take a village to raise a child. 